\title{
Roy R. Casiano: Endoscopic sinonasal dissection guide
}

\section{Thieme verlag, ISBN: 978-1-60406-587-9}

\section{Arild Danielsen}

Received: 28 February 2012 / Accepted: 6 March 2012 / Published online: 21 April 2012

(C) Springer-Verlag 2012

The aim of this book, which also includes a video of a surgical case, is to show important procedures in endoscopic step-by-step dissection in the cadaver laboratory.

The result is a book containing six chapters starting with an introduction to endoscopic sinonasal surgery, discussing instrumentation and patient positioning, considering anteroposterior versus posteroanterior approach, the importance of focusing on anatomical landmarks, basic endoscopic sinonasal dissection and advanced endoscopic sinonasal dissection. The latter involving extended approaches to the orbit, optic nerve, pterygomaxillary fossa and anterior skull base.
The illustrations are all of high quality. There is an introduction to each chapter and a special focus on vital landmarks. An extended reference list contains an update on important studies published within the field of endoscopic endonasal surgery.

This book is considered of vital importance both to residents and experienced surgeons and is hereby highly recommended.

Bergen, February 23th 2012

\footnotetext{
A. Danielsen ( $\square)$

Department of Otorhinolaryngology/Head and Neck Surgery,

Haukeland University Hospital, 5021 Bergen, Norway

e-mail: arild.danielsen@helse-bergen.no
} 